\title{
I diopathic reversible cerebral vasoconstriction syndrome in a female patient complicated by subarachnoid haemorrhage: A case report
}

\author{
Joga Chaganti, Pramod Phadke, Hyunmin Park, Manish Chug \\ Nepean Hospital and Blue Mountains Local Health District, Sydney, Australia \\ Correspondence: Joga Chaganti. Address: St Vincent's Hospital, Sydney, Australia. \\ Email: Joga.Chaganti@svha.org.au
}

Received: November 17, 2014

Accepted: December 21, 2014 Online Published: March 5, 2015

DOI : $10.5430 /$ ijdi.v2n2p11

URL: http://dx.doi.org/10.5430/ijdi.v2n2p11

\begin{abstract}
Introduction: RCVS is a cerebrovascular disorder characterized by multisegmental narrowing of the cerebral arteries with a spontaneous resolution in 1-3 months. It most commonly presents as a sudden onset of a "thunderclap" headache which may be associated with mild neurological symptoms. It may be complicated by subarachnoid haemorrhage, intracerebral haemorrhage, stroke or seizures. Diagnosis is confirmed by "string of beads appearance" of the intracerebral arteries on a cerebral angiography.

Case presentation: We present a case of a 65-year-old female, who presented with sudden onset of intense headache and associated with visual disturbances (homonymous hemianopia) with CT evidence of subarachnoid haemorrhage in the superficial cortical sulci. Catheter angiography showed string of beads pattern with focal areas of vasoconstriction and dilatation. Provisional diagnosis of RCVS was made and the patient was treated with calcium channel blockers. Patient demonstrated complete resolution of symptoms and got discharged.

Conclusion: RCVS is being increasingly recognised as one of the causes of thunderclap headaches and demonstrates characteristic abnormalities on angiography which are reversible over time. High degree of suspicion and timely diagnosis can potentially reduce the morbidity.
\end{abstract}

\section{Keywords}

Reversible cerebral vasoconstriction syndrome, Headache, Subarachnoid haemorrhage

\section{I ntroduction}

RCVS is characterised by a sudden severe headache, multiple segmental narrowing of the cerebral arteries and lack of aneurismal subarachnoid haemorrhage, near normal CSF and spontaneous resolution of arterial narrowing within 3 months ${ }^{[1]}$. Imaging findings in RCVS are varied and could be associated with non-aneurismal SAH, ischaemic or haemorrhagic stroke, RCVS is more commonly seen in middle aged women. The angiographic findings are similar to the other vasculopathies and show multifocal areas of vascular narrowing. However these segmental, focal areas of narrowing are reversible and usually resolve in few months. Treatment is usually with calcium channel blockers and discontinuation 
of the offending drugs, such as exposure to vasoactive substance mainly cannabis, selective serotonin reuptake inhibitors, and use of nasal decongestants. Corticosteroids are typically contraindicated.

\section{Case presentation}

A 65-year Caucasian female patient was referred with 1 week history of recurrent severe generalised headache associated with right homonymous hemianopia. She had no other relevant neurological findings on a clinical examination. Patient's past history included hypercholesterolaemia and hypothyroidism. She was taking $125 \mathrm{mcg}$ of thyroxine every day for hypothyroidism and her thyroid function tests were normal at the time of admission (TSH was $1.15 \mathrm{mlU} / \mathrm{L}$ and free thyroxine level was $17.7 \mu \mathrm{mol} / \mathrm{L}$ ). She was not on any statins at the time of admission and her fasting serum cholesterol, LDL and triglyeride levels were elevated (Cholesterol - $6.4 \mathrm{mmol} / \mathrm{L}$, LDL $-4.2 \mathrm{mmol} / \mathrm{L}$ and Triglycerides $2.16 \mathrm{mmol} / \mathrm{L}$ ). She was subsequently commenced on $40 \mathrm{mg} /$ day of oral Atorvastatin by the treating team.

A CT brain demonstrated a subarachnoid haemorrhage within the right frontal and left occipital lobes surface CSF spaces (see Figure 1). MR Angiography showed suspicious focal areas of arterial irregularity in the anterior circulation, besides confirming SAH in the superficial sulcal spaces (see Figure 2). A digital subtraction angiography of the intracerebral vessels demonstrated multisegmental vasoconstriction of the intracerebral vessels with typical "string of beads" appearance within both anterior and posterior circulation (see Figure 3). Patient was commenced on oral Nimodipine (30 mg every 4 hours) by the treating team. A follow up CT brain prior to the discharge showed clearance of SAH (see Figure 4). Patient was discharged on oral Nimodipine, nine days after her initial presentation and her symptoms had completely resolved.

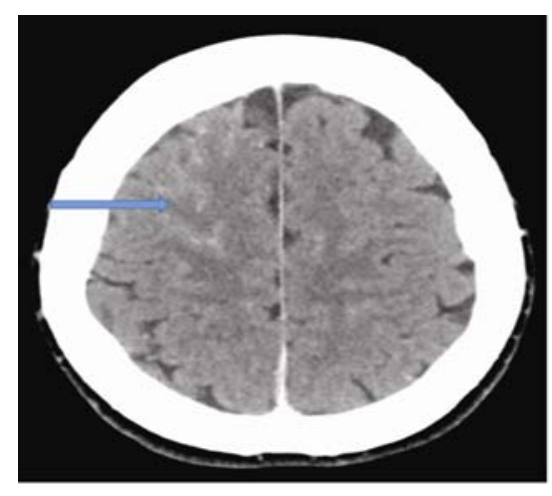

Figure 1. Axial CT showing hyperdense sulci in the right high frontal region indicating blood in the subarachnoid spaces(SAH)
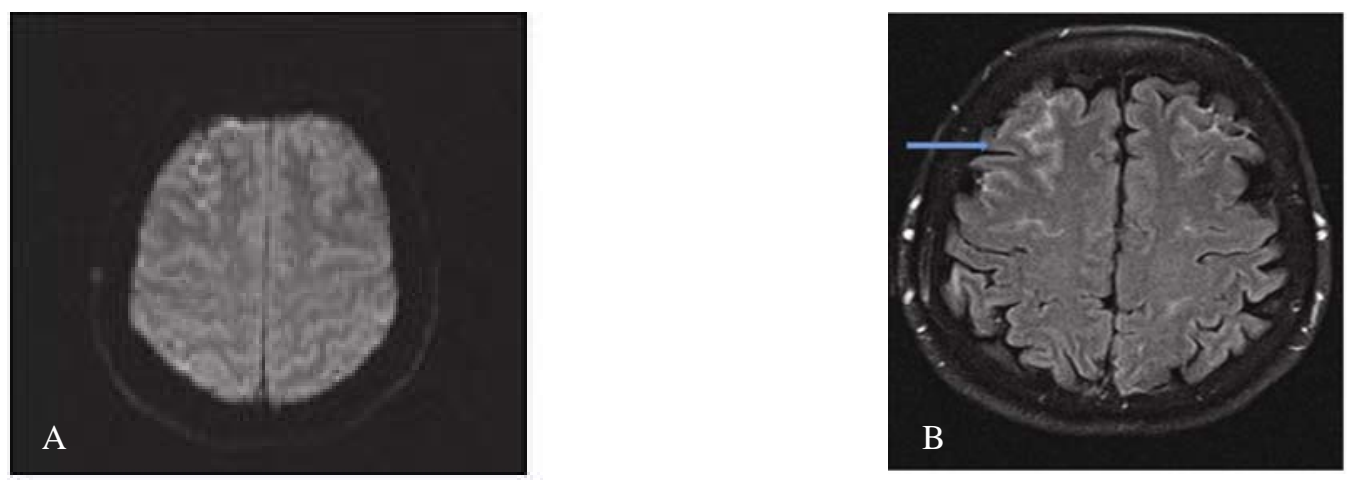

Figure 2. MR BRAIN -Axial GRE and FLAIR sequences showing hyperintense sulcal spaces suggestive of haemorrhage 

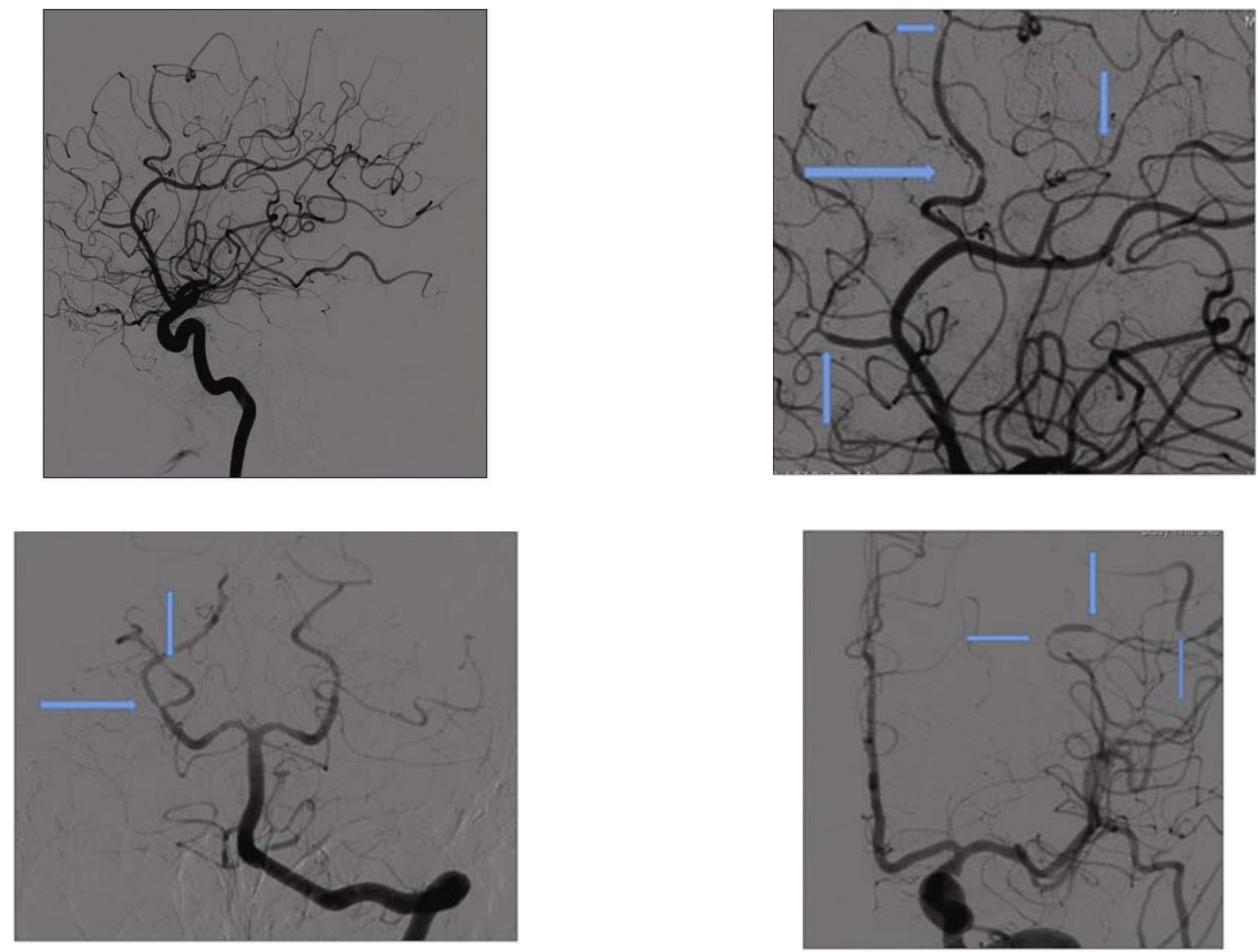

Figure 3. Digital subtraction angiography showing multi focal areas of narrowing and dilatation (Arrows) involving both the anterior circulation and posterior circulation arteries
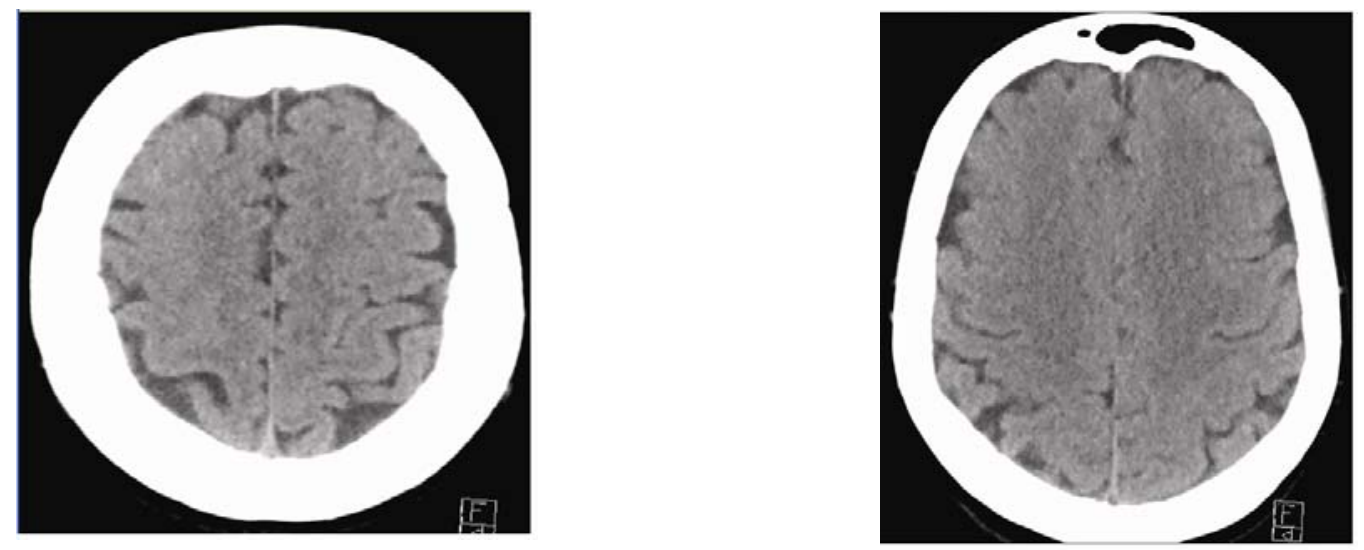

Figure 4. Repeat CT after 1 week demonstrating clearance of SAH

\section{Discussion}

RCVS is increasingly recognized due to wider availability of the imaging techniques. Sattar et al. ${ }^{[1]}$ in their meta-analysis identified that there were total of 214 patients reported in the literature with RCVS (four case series and rest are case reports). Although the pathophysiology was not clearly understood, disturbance in the control of cerebrovascular tone appears to be the final common pathway. A prospective study ${ }^{[2]}$ of 67 patients with RCVS observed over 3 year period reported that the main pattern of presentation (94\%) was recurrent thunder clap headache. The criteria standard for the diagnosis is angiography which reveals string of beads appearance secondary to alternating areas of vascular narrowing and dilatation. Vascular abnormalities can be identified with MRA, CTA and TCD. The imaging findings of RCVS are 
similar to PRES ${ }^{[5]}$ and PACNS. PRES demonstrate vasogenic oedema, range of diffusion abnormalities, isolated SAH as well as vasculopathy with string of beads appearance involving second and third order arteries. Indeed many consider the pathophysiology of RCVS and PRES are similar ${ }^{[3]}$. The second group of disorders which can closely resemble RCVS is PACNS ${ }^{[4]}$. However the clinical course of PACNS is significantly different and untreated can lead to progressive neurological impairment and death.

\section{Conclusion}

The diagnosis of RCVS requires high degree of suspicion and timely diagnosis can potentially minimise the complications. Digital subtraction angiography (DSA) has a better spatial resolution than MRA and CTA for medium and smaller sized intra cranial arteries and therefore subtle irregularities in cerebral vasculature are better identified by angiography. This makes digital subtraction cerebral angiography the criteria standard for the diagnosis of RCVS.

\section{References}

[1] Sattar A, Manousakis G, Jensen MB. Systematic review of reversible cerebral vasoconstriction syndrome. Expert Rev Cardiovasc Ther. 2010; 8(10): 1417-1421. PMid:20936928 http://dx.doi.org/10.1586/erc.10.124

[2] Ducros A, Boukobza M, Porcher R, et al. The clinical and radiological spectrum of reversible cerebral vasoconstriction syndrome. A prospective series of 67 patients. Brain. 2007; 130(12): 3091-3101. PMid:18025032 http://dx.doi.org/10.1093/brain/awm256

[3] Chen SP, Fuh JL, Wang SJ. Magnetic resonance angiography in reversible cerebral vasoconstriction syndromes. Annals of Neurology. 2010; 67: 648-56. PMid:20437562

[4] Calabrese LH, Dodick DW, Schwedt TJ, et al. Narrative review: reversible cerebral vasoconstriction syndromes. Annals of Internal Medicine. 2007; 146(1): 36-44. http://dx.doi.org/10.7326/0003-4819-146-1-200701020-00007

[5] Marder CP, Donohue MM, Weinstein JR, et al. Multimodal Imaging of Reversible Cerebral Vasoconstriction Syndrome: A Series of 6 Cases. American journal of Neuroradiology. 2012; 33: 1403-1411. PMid:22422190 http://dx.doi.org/10.3174/ajnr.A2964 